\title{
An artificial proof of a geometric inequality in a triangle
}

\author{
Shi-Chang Shi ${ }^{{ }^{*}}$ and Yu-Dong $\mathrm{Wu}^{2}$
}

"Correspondence:

532686108@qq.com

1 Department of Education, Zhejiang Teaching and Research Institute, Hangzhou, Zhejiang 310012, People's Republic of China Full list of author information is available at the end of the article

\begin{abstract}
In this paper, the authors give an artificial proof of a geometric inequality relating to the medians and the exradius in a triangle by making use of certain analytical techniques for systems of nonlinear algebraic equations.
\end{abstract}

MSC: $51 \mathrm{M16} ; 52 \mathrm{~A} 40$

Keywords: geometric inequality; triangle; medians; inradius; circumradius

\section{Introduction and main results}

For a given $\triangle A B C$, let $a, b$ and $c$ denote the side-lengths facing the angles $A, B$ and $C$, respectively. Also, let $m_{a}, m_{b}$ and $m_{c}$ denote the corresponding medians, $r_{a}, r_{b}$ and $r_{c}$ the corresponding exradii, $s=\frac{1}{2}(a+b+c)$ the semi-perimeter, $\Delta$ the area. In addition, we let

$$
\begin{aligned}
& m_{1}=\frac{1}{2} \sqrt{(b+c)^{2}-a^{2}}=\sqrt{s(s-a)} \\
& m_{2}=\frac{1}{2} \sqrt{2 a^{2}+\frac{1}{4}(b+c)^{2}}
\end{aligned}
$$

and

$$
r_{1}=\frac{a \sqrt{s(s-a)}}{2(s-a)} .
$$

Throughout this paper, we will customarily use the cyclic sum symbols as follows:

$$
\sum f(a)=f(a)+f(b)+f(c)
$$

and

$$
\sum f(b, c)=f(a, b)+f(b, c)+f(c, a) .
$$

In 2003, Liu [1] found the following interesting geometric inequality relating to the medians and the exradius in a triangle with the computer software BOTTEMA invented by Yang [2-5], and Liu thought this inequality cannot be proved by a human.

Theorem 1.1 In $\triangle A B C$, the best constant $k$ for the following inequality

$$
\sum\left(r_{b}-r_{c}\right)^{2} \geq k \cdot \sum\left(m_{b}-m_{c}\right)^{2}
$$

\section{照 Springer}

C 2013 Shi and Wu; licensee Springer. This is an Open Access article distributed under the terms of the Creative Commons Attribution License (http://creativecommons.org/licenses/by/2.0), which permits unrestricted use, distribution, and reproduction in any medium, provided the original work is properly cited. 
is the real root on the interval $(3,4)$ of the following equation

$$
6,561 k^{4}-14,256 k^{3}-18,080 k^{2}-25,344 k+20,736=0 .
$$

Furthermore, the constant $k$ has its numerical approximation given by 3.2817755127 .

In this paper, the authors give an artificial proof of Theorem 1.1.

\section{Preliminary results}

In order to prove Theorem 1.1, we require the following results.

Lemma 2.1 In $\triangle A B C$, if $a \leq b \leq c$, then

$$
r_{a}^{2}+r_{b}^{2}+r_{c}^{2}-\left(r_{1}^{2}+2 m_{1}^{2}\right) \geq \frac{3 s(s-a)(b-c)^{2}}{4(s-b)(s-c)}
$$

Proof From $a=(s-b)+(s-c)$ and the formulas of the exradius $r_{a}=\frac{\Delta}{s-a}=\frac{\sqrt{s(s-a)(s-b)(s-c)}}{s-a}$, etc., we get

$$
\begin{aligned}
r_{a}^{2} & +r_{b}^{2}+r_{c}^{2}-\left(r_{1}^{2}+2 m_{1}^{2}\right) \\
& =\left[\frac{1}{(s-a)^{2}}+\frac{1}{(s-b)^{2}}+\frac{1}{(s-c)^{2}}\right] s(s-a)(s-b)(s-c)-\frac{a^{2} s(s-a)}{4(s-a)^{2}}-2 s(s-a) \\
& =\frac{1}{4} s(s-a)\left[\frac{4(s-b)(s-c)}{(s-a)^{2}}+\frac{4(s-b)(s-c)}{(s-b)^{2}}+\frac{4(s-b)(s-c)}{(s-c)^{2}}-\frac{a^{2}}{(s-a)^{2}}-8\right] \\
& =\frac{1}{4} s(s-a)\left[\frac{4(s-b)(s-c)-a^{2}}{(s-a)^{2}}+4\left(\frac{s-c}{s-b}+\frac{s-b}{s-c}-2\right)\right] \\
& =\frac{1}{4} s(s-a)\left[-\frac{(b-c)^{2}}{(s-a)^{2}}+\frac{4(b-c)^{2}}{(s-b)(s-c)}\right] \\
& =\frac{1}{4} s(s-a)(b-c)^{2}\left[\frac{4}{(s-b)(s-c)}-\frac{1}{(s-a)^{2}}\right] .
\end{aligned}
$$

For $a \leq b \leq c$, we have

$$
s-a \geq s-b \geq s-c>0,
$$

then

$$
0<\frac{1}{s-a} \leq \frac{1}{s-b} \leq \frac{1}{s-c}
$$

hence

$$
\frac{1}{(s-b)(s-c)} \geq \frac{1}{(s-a)^{2}}>0 .
$$

Inequality (2.1) follows from inequalities (2.2)-(2.3) immediately. 
Lemma 2.2 In $\triangle A B C$, we have

$$
\left(m_{b}+m_{2}\right)\left(m_{c}+m_{2}\right) \geq 4 s \sqrt{(s-b)(s-c)}
$$

and

$$
a\left(m_{b}+m_{c}\right)^{2}-8 s(s-b)(s-c) \geq \frac{3 s \sqrt{(s-b)(s-c)}(b-c)^{2}}{a} .
$$

Proof of inequality (2.4) From

$$
m_{2}^{2}-\frac{1}{2} a s=\frac{1}{4}\left(a-\frac{b+c}{2}\right)^{2} \geq 0
$$

we immediately obtain

$$
m_{2} \geq \sqrt{\frac{1}{2} a s}
$$

In view of the $A M-G M$ inequality, we get

$$
\frac{a}{2}=\frac{(s-b)+(s-c)}{2} \geq \sqrt{(s-b)(s-c)} .
$$

By the power mean inequality, we have

$$
\sqrt{\frac{a}{2}}=\sqrt{\frac{(s-b)+(s-c)}{2}} \geq \frac{\sqrt{s-b}+\sqrt{s-c}}{2} .
$$

By the well-known inequalities $m_{b} \geq \sqrt{s(s-b)}$ and $m_{c} \geq \sqrt{s(s-c)}$, together with inequalities (2.6)-(2.8), we obtain

$$
\begin{aligned}
& \left(m_{b}+m_{2}\right)\left(m_{c}+m_{2}\right) \\
& \geq\left(\sqrt{s(s-b)}+\sqrt{\frac{1}{2} a s}\right)\left(\sqrt{s(s-c)}+\sqrt{\frac{1}{2} a s}\right) \\
& =s\left(\sqrt{s-b}+\sqrt{\frac{1}{2} a}\right)\left(\sqrt{s-c}+\sqrt{\frac{1}{2} a}\right) \\
& =s\left[\frac{1}{2} a+\sqrt{\frac{1}{2} a}(\sqrt{s-b}+\sqrt{s-c})+\sqrt{(s-b)(s-c)}\right] \\
& \geq s\left[\frac{1}{2}(\sqrt{s-b}+\sqrt{s-c})^{2}+2 \sqrt{(s-b)(s-c)}\right] \\
& =s\left[\frac{1}{2} a+3 \sqrt{(s-b)(s-c)}\right] \\
& \geq 4 s \sqrt{(s-b)(s-c)} .
\end{aligned}
$$

The proof of inequality (2.4) is thus complete. 
Proof of inequality (2.5) According to the well-known inequalities $m_{b} \geq \sqrt{s(s-b)}, m_{c} \geq$ $\sqrt{s(s-c)}$ and inequality (2.7), we have

$$
\begin{aligned}
a\left(m_{b}\right. & \left.+m_{c}\right)^{2}-8 s(s-b)(s-c) \\
= & {[a-2 \sqrt{(s-b)(s-c)}]\left(m_{b}+m_{c}\right)^{2} } \\
& +2 \sqrt{(s-b)(s-c)}\left[\left(m_{b}+m_{c}\right)^{2}-4 s \sqrt{(s-b)(s-c)}\right] \\
\geq & {[a-2 \sqrt{(s-b)(s-c)}] \cdot 4 m_{b} m_{c}+2 \sqrt{(s-b)(s-c)}\left[(\sqrt{s(s-b)}+\sqrt{s(s-c)})^{2}\right.} \\
& -4 s \sqrt{(s-b)(s-c)}] \\
\geq & 4 s[a-2 \sqrt{(s-b)(s-c)}] \sqrt{(s-b)(s-c)}+2 \sqrt{(s-b)(s-c)}[a-2 \sqrt{(s-b)(s-c)}] \\
= & 6 s \sqrt{(s-b)(s-c)}[a-2 \sqrt{(s-b)(s-c)}] \\
= & \frac{6 s \sqrt{(s-b)(s-c)}(b-c)^{2}}{a+2 \sqrt{(s-b)(s-c)}} \\
\geq & \frac{3 s \sqrt{(s-b)(s-c)(b-c)^{2}}}{a} .
\end{aligned}
$$

Hence, we complete the proof of inequality (2.5).

Lemma 2.3 In $\triangle A B C$, we have

$$
m_{b} m_{c} \leq m_{2}^{2}
$$

Proof From the formulas of the medians, we have

$$
\begin{aligned}
m_{b} m_{c}-m_{2}^{2} & =\frac{m_{b}^{2} m_{c}^{2}-m_{2}^{4}}{m_{b} m_{c}+m_{2}^{2}} \\
& =\frac{\frac{1}{16}\left(2 c^{2}+2 a^{2}-b^{2}\right)\left(2 a^{2}+2 b^{2}-c^{2}\right)-\frac{1}{16}\left(2 a^{2}+\frac{1}{4}(b+c)^{2}\right)}{m_{b} m_{c}+m_{2}^{2}} \\
& =\frac{\left\{16\left[a^{2}-(b+c)^{2}\right]-\left(17 b^{2}+17 c^{2}+38 b c\right)\right\}(b-c)^{2}}{256\left(m_{b} m_{c}+m_{2}^{2}\right)} \leq 0 .
\end{aligned}
$$

Therefore, inequality (2.10) holds true.

Lemma 2.4 In $\triangle A B C$, if $a \leq b \leq c$, then

$$
\begin{aligned}
& \frac{m_{b}+m_{c}}{m_{a}+m_{1}}+\frac{1}{4}\left(\frac{m_{1}}{m_{2}+m_{b}}+\frac{m_{1}}{m_{2}+m_{c}}\right)-\frac{9(b+c)^{2}}{8\left(m_{b}+m_{c}\right)^{2}} \\
& \geq \frac{m_{2}}{m_{1}}+\frac{m_{1}}{4 m_{2}}-\frac{9 a(b+c)^{2}}{64 s(s-b)(s-c)} .
\end{aligned}
$$

Proof It is obvious that $m_{b}>c-\frac{b}{2}$ and $m_{c}>b-\frac{c}{2}$, then we have $m_{b}+m_{c}>\frac{1}{2}(b+c)$, thus

$$
\left(m_{b}-m_{c}\right)^{2}=\frac{\left(m_{b}^{2}-m_{c}^{2}\right)^{2}}{\left(m_{b}+m_{c}\right)^{2}}=\frac{9(b+c)^{2}(b-c)^{2}}{16\left(m_{b}+m_{c}\right)^{2}} \leq \frac{9}{4}(b-c)^{2} .
$$


For $a \leq b \leq c$, we have that

$$
m_{a} \geq\left\{\begin{array}{l}
m_{1} \\
m_{b}
\end{array} \geq m_{2} \geq m_{c} .\right.
$$

By Lemma 2.3 and inequalities (2.12)-(2.13), we have

$$
\begin{aligned}
\frac{m_{b}}{m_{a}}+m_{c} & +\frac{1}{4}\left(\frac{m_{1}}{m_{2}+m_{b}}+\frac{m_{1}}{m_{2}+m_{c}}\right)-\frac{m_{2}}{m_{1}}-\frac{m_{1}}{4 m_{2}} \\
& =\frac{m_{b}+m_{c}-2 m_{2}}{m_{a}+m_{1}}+\frac{m_{2}\left(m_{1}-m_{a}\right)}{m_{1}\left(m_{a}+m_{1}\right)}+\frac{m_{1}\left(m_{2}^{2}-m_{b} m_{c}\right)}{4 m_{2}\left(m_{2}+m_{b}\right)\left(m_{2}+m_{c}\right)} \\
& \geq \frac{\left(m_{b}+m_{c}\right)^{2}-4 m_{2}^{2}}{\left(m_{a}+m_{1}\right)\left(m_{b}+m_{c}+2 m_{2}\right)}+\frac{m_{2}\left(m_{1}^{2}-m_{a}^{2}\right)}{m_{1}\left(m_{a}+m_{1}\right)^{2}} \\
& =\frac{2\left(m_{b}^{2}+m_{c}^{2}\right)-\left(m_{b}-m_{c}\right)^{2}-4 m_{2}^{2}}{\left(m_{a}+m_{1}\right)\left(m_{b}+m_{c}+2 m_{2}\right)}+\frac{m_{2}\left(m_{1}^{2}-m_{a}^{2}\right)}{m_{1}\left(m_{a}+m_{1}\right)^{2}} \\
& =\frac{\frac{1}{4}(b-c)^{2}-\left(m_{b}-m_{c}\right)^{2}}{\left(m_{a}+m_{1}\right)\left(m_{b}+m_{c}+2 m_{2}\right)}-\frac{m_{2}(b-c)^{2}}{4 m_{1}\left(m_{a}+m_{1}\right)^{2}} \\
& \geq \frac{\frac{1}{4}(b-c)^{2}-\frac{9}{4}(b-c)^{2}}{\left(m_{a}+m_{1}\right)\left(m_{b}+m_{c}+2 m_{2}\right)}-\frac{(b-c)^{2}}{4\left(m_{a}+m_{1}\right)^{2}} \\
& =\frac{-2(b-c)^{2}}{\left(m_{a}+m_{1}\right)\left(m_{b}+m_{c}+2 m_{2}\right)}-\frac{(b-c)^{2}}{4\left(m_{a}+m_{1}\right)^{2}} \\
& \geq \frac{-2(b-c)^{2}}{\left(m_{a}+m_{1}\right)\left(m_{b}+m_{c}\right)}-\frac{(b-c)^{2}}{4\left(m_{a}+m_{1}\right)^{2}} \\
& =\frac{-2(b-c)^{2}}{\left(m_{b}+m_{c}\right)^{2}-\frac{(b-c)^{2}}{4\left(m_{b}+m_{c}\right)^{2}}} \\
& \frac{-9(b-c)^{2}}{4 m^{2}} \cdot \\
&
\end{aligned}
$$

By inequality (2.5), (2.7) and $a \leq b \leq c$, we obtain that

$$
\begin{aligned}
& \frac{9 a(b+c)^{2}}{64 s(s-b)(s-c)}-\frac{9(b+c)^{2}}{8\left(m_{b}+m_{c}\right)^{2}} \\
& =\frac{9(b+c)^{2}\left[a\left(m_{b}+m_{c}\right)^{2}-8 s(s-b)(s-c)\right]}{64 s(s-b)(s-c)\left(m_{b}+m_{c}\right)^{2}} \\
\geq & \frac{9(b+c)^{2}}{64 s(s-b)(s-c)\left(m_{b}+m_{c}\right)^{2}} \cdot \frac{3 s \sqrt{(s-b)(s-c)}(b-c)^{2}}{a} \\
= & \frac{27(b+c)^{2}(b-c)^{2}}{64 a \sqrt{(s-b)(s-c)}\left(m_{b}+m_{c}\right)^{2}} \\
\geq & \frac{27(b+c)^{2}(b-c)^{2}}{32 a^{2}\left(m_{b}+m_{c}\right)^{2}} \\
\geq & \frac{27(b-c)^{2}}{8\left(m_{b}+m_{c}\right)^{2}} .
\end{aligned}
$$


By inequalities (2.14)-(2.15), we have

$$
\begin{aligned}
& {\left[\frac{m_{b}+m_{c}}{m_{a}+m_{1}}+\frac{1}{4}\left(\frac{m_{1}}{m_{2}+m_{b}}+\frac{m_{1}}{m_{2}+m_{c}}\right)-\frac{9(b+c)^{2}}{8\left(m_{b}+m_{c}\right)^{2}}\right]} \\
& \quad-\left[\frac{m_{2}}{m_{1}}+\frac{m_{1}}{4 m_{2}}-\frac{9 a(b+c)^{2}}{64 s(s-b)(s-c)}\right] \\
& =\left[\frac{m_{b}+m_{c}}{m_{a}+m_{1}}+\frac{1}{4}\left(\frac{m_{1}}{m_{2}+m_{b}}+\frac{m_{1}}{m_{2}+m_{c}}\right)-\frac{m_{2}}{m_{1}}-\frac{m_{1}}{4 m_{2}}\right] \\
& \quad+\left[\frac{9 a(b+c)^{2}}{64 s(s-b)(s-c)}-\frac{9(b+c)^{2}}{8\left(m_{b}+m_{c}\right)^{2}}\right] \\
& \geq \frac{-9(b-c)^{2}}{4\left(m_{b}+m_{c}\right)^{2}}+\frac{27(b-c)^{2}}{8\left(m_{b}+m_{c}\right)^{2}} \\
& =\frac{9(b-c)^{2}}{8\left(m_{b}+m_{c}\right)^{2}} \geq 0 .
\end{aligned}
$$

Inequality (2.11) follows from inequality (2.16) immediately.

Lemma 2.5 In $\triangle A B C$, if $a \leq b \leq c$, then

$$
\frac{m_{2}}{m_{1}}+\frac{m_{1}}{4 m_{2}}+\frac{3(b+c)^{2}}{16 a^{2}} \geq 2
$$

and

$$
\frac{m_{1}+\sqrt{3} a}{s} \leq \sqrt{3} \text {. }
$$

Proof Without loss of generality, we can take $b+c=2$ and $a=x$, for $a \leq b \leq c$, we have $0<x \leq 1$.

(i) First, we prove inequality (2.17).

$$
\begin{aligned}
\frac{m_{2}}{m_{1}}+\frac{m_{1}}{4 m_{2}}+\frac{3(b+c)^{2}}{16 a^{2}}-2 & =\sqrt{\frac{1+2 x^{2}}{4-x^{2}}}+\frac{1}{4} \sqrt{\frac{4-x^{2}}{1+2 x^{2}}}+\frac{3}{4 x^{2}}-2 \\
& =\frac{8+7 x^{2}}{4 \sqrt{\left(4-x^{2}\right)\left(1+2 x^{2}\right)}}+\frac{3\left(1-x^{2}\right)}{4 x^{2}}-\frac{5}{4} \\
& \geq \frac{8+7 x^{2}}{4 \cdot \frac{\left(4-x^{2}\right)+\left(1+2 x^{2}\right)}{2}}+\frac{3\left(1-x^{2}\right)}{4 x^{2}}-\frac{5}{4} \\
& =\frac{8+7 x^{2}}{2\left(5+x^{2}\right)}+\frac{3\left(1-x^{2}\right)}{4 x^{2}}-\frac{5}{4} \\
& =\frac{9\left(x^{2}-1\right)}{4\left(5+x^{2}\right)}+\frac{3\left(1-x^{2}\right)}{4 x^{2}} \\
& \geq \frac{3\left(x^{2}-1\right)}{8}+\frac{3\left(1-x^{2}\right)}{4} \\
& =\frac{3\left(1-x^{2}\right)}{8} \geq 0 .
\end{aligned}
$$

Inequality (2.19) terminates the proof of inequality (2.17). 
(ii) Second, we prove inequality (2.18).

$$
\begin{aligned}
m_{1} & +\sqrt{3} a-\sqrt{3} s \\
& =\frac{1}{2} \sqrt{4-x^{2}}-\frac{\sqrt{3}}{2}(2-x) \\
& =\frac{1}{2} \sqrt{2-x}(\sqrt{2+x}-\sqrt{3(2-x)}) \\
& =\frac{-2 \sqrt{2-x}(1-x)}{\sqrt{2+x}+\sqrt{3(2-x)}} \leq 0 .
\end{aligned}
$$

Inequality (2.18) follows from inequality (2.20) immediately.

Lemma 2.6 In $\triangle A B C$, if $a \leq b \leq c$, then

$$
m_{a} m_{b}+m_{b} m_{c}+m_{c} m_{a}-2 m_{1} m_{2}-m_{2}^{2} \geq \frac{3}{8}(b-c)^{2}-\frac{3 s(s-a)(b-c)^{2}}{16(s-b)(s-c)} .
$$

Proof By the $A M-G M$ inequality, the well-known inequalities $m_{b} \geq \sqrt{s(s-b)}$ and $m_{c} \geq$ $\sqrt{s(s-c)}$, we get

$$
\left(m_{b}+m_{c}\right)^{2} \geq 4 m_{b} m_{c} \geq 4 s \sqrt{(s-b)(s-c)} \geq 6 a \sqrt{(s-b)(s-c)} \geq 12(s-b)(s-c)
$$

or

$$
m_{b}+m_{c} \geq 2 \sqrt{3} \sqrt{(s-b)(s-c)} .
$$

By inequalities (2.4), (2.10), (2.11), (2.17), (2.22), we obtain that

$$
\begin{aligned}
& m_{a} m_{b}+m_{b} m_{c}+m_{c} m_{a}-2 m_{1} m_{2}-m_{2}^{2} \\
&=\frac{\left(m_{b}+m_{c}\right)\left(m_{a}^{2}-m_{1}^{2}\right)}{m_{a}+m_{1}}+\frac{m_{1}\left(m_{b}^{2}-m_{2}^{2}\right)}{m_{b}+m_{2}}+\frac{m_{1}\left(m_{c}^{2}-m_{2}^{2}\right)}{m_{c}+m_{2}}-\frac{\left(m_{b}^{2}-m_{c}^{2}\right)^{2}}{2\left(m_{b}+m_{c}\right)^{2}}+\frac{1}{16}(b-c)^{2} \\
&=\frac{\left(m_{b}+m_{c}\right)(b-c)^{2}}{4\left(m_{a}+m_{1}\right)}+\frac{m_{1}(5 b+7 c)(c-b)}{16\left(m_{b}+m_{2}\right)}+\frac{m_{1}(7 b+5 c)(b-c)}{16\left(m_{c}+m_{2}\right)} \\
& \quad-\frac{9(b+c)^{2}(b-c)^{2}}{32\left(m_{b}+m_{c}\right)^{2}}+\frac{1}{16}(b-c)^{2} \\
&=\frac{\left(m_{b}+m_{c}\right)(b-c)^{2}}{4\left(m_{a}+m_{1}\right)}+\frac{m_{1}(b-c)^{2}}{16\left(m_{b}+m_{2}\right)}+\frac{m_{1}(b-c)^{2}}{16\left(m_{c}+m_{2}\right)} \\
& \quad-\frac{9 m_{1}(b+c)^{2}(b-c)^{2}}{32\left(m_{b}+m_{2}\right)\left(m_{c}+m_{2}\right)\left(m_{b}+m_{c}\right)} \\
& \quad-\frac{9(b+c)^{2}(b-c)^{2}}{32\left(m_{b}+m_{c}\right)^{2}}+\frac{1}{16}(b-c)^{2} \\
& \geq \frac{1}{4}\left(\frac{m_{2}}{m_{1}}+\frac{m_{1}}{4 m_{2}}-\frac{9 a(b+c)^{2}}{64 s(s-b)(s-c)}-\frac{m_{1}(b+c)^{2}}{64 \sqrt{3} s(s-b)(s-c)}+\frac{1}{4}\right)(b-c)^{2} \\
&= \frac{1}{4}\left(\frac{m_{2}}{m_{1}}+\frac{m_{1}}{4 m_{2}}-\frac{9\left(m_{1}+\sqrt{3} a\right)(b+c)^{2}}{64 \sqrt{3} s(s-b)(s-c)}+\frac{1}{4}\right)(b-c)^{2}
\end{aligned}
$$


Sh and Wu Journal of Inequalities and Applications 2013, 2013:329

Page 8 of 13

http://www.journalofinequalitiesandapplications.com/content/2013/1/329

$$
\begin{aligned}
& \geq \frac{1}{4}\left(\frac{m_{2}}{m_{1}}+\frac{m_{1}}{4 m_{2}}-\frac{9(b+c)^{2}}{64(s-b)(s-c)}+\frac{1}{4}\right)(b-c)^{2} \\
& =\frac{1}{4}\left(\frac{m_{2}}{m_{1}}+\frac{m_{1}}{4 m_{2}}-\frac{3\left[(b+c)^{2}-a^{2}\right]}{16(s-b)(s-c)}+\frac{3\left[(b+c)^{2}-4 a^{2}\right]}{64(s-b)(s-c)}+\frac{1}{4}\right)(b-c)^{2} \\
& \geq \frac{1}{4}\left(\frac{m_{2}}{m_{1}}+\frac{m_{1}}{4 m_{2}}-\frac{3 s(s-a)}{4(s-b)(s-c)}+\frac{3\left[(b+c)^{2}-4 a^{2}\right]}{16 a^{2}}+\frac{1}{4}\right)(b-c)^{2} \\
& =\frac{1}{4}\left(\frac{m_{2}}{m_{1}}+\frac{m_{1}}{4 m_{2}}+\frac{3(b+c)^{2}}{16 a^{2}}-\frac{3 s(s-a)}{4(s-b)(s-c)}-\frac{1}{2}\right)(b-c)^{2} \\
& \geq \frac{1}{4}\left(2-\frac{3 s(s-a)}{4(s-b)(s-c)}-\frac{1}{2}\right)(b-c)^{2} \\
& =\frac{3}{8}(b-c)^{2}-\frac{3 s(s-a)(b-c)^{2}}{16(s-b)(s-c)}
\end{aligned}
$$

The proof of Lemma 2.6 is thus completed.

Lemma 2.7 In $\triangle A B C$, if inequality (1.1) holds, then $k \leq 4$.

Proof Let $b=c=1$ and $a=x$. For $a \leq b \leq c$, we have $x \in(0,1]$, then inequality (1.1) is equivalent to

$$
\begin{gathered}
2\left(\frac{x \sqrt{4-x^{2}}}{2(2-x)}-\frac{\sqrt{4-x^{2}}}{2}\right)^{2} \geq 2 k\left(\frac{\sqrt{4-x^{2}}}{2}-\frac{\sqrt{2 x^{2}+1}}{2}\right)^{2} \\
\Longleftrightarrow \quad \frac{2+x}{2-x} \geq k \cdot \frac{9(1+x)^{2}}{4\left(\sqrt{4-x^{2}}+\sqrt{2 x^{2}+1}\right)^{2}} \\
\Longleftrightarrow \quad k \leq \frac{4(2+x)\left(\sqrt{4-x^{2}}+\sqrt{2 x^{2}+1}\right)^{2}}{9(2-x)(1+x)^{2}} .
\end{gathered}
$$

Taking $x=1$ in inequality (2.23), we obtain that $k \leq 4$.

Lemma 2.8 In $\triangle A B C$, if $a \leq b \leq c$ and $0<k \leq 4$, then we have

$$
\sum\left(r_{b}-r_{c}\right)^{2}-k \cdot \sum\left(m_{b}-m_{c}\right)^{2} \geq 2\left(r_{1}-m_{1}\right)^{2}-2 k\left(m_{1}-m_{2}\right)^{2} .
$$

Proof For

$$
\sum\left(r_{b}-r_{c}\right)^{2}=2 \sum r_{a}^{2}-2 \sum r_{b} r_{c}=2 \sum r_{a}^{2}-2 s^{2}
$$

and

$$
\sum\left(m_{b}-m_{c}\right)^{2}=2 \sum m_{a}^{2}-2 \sum m_{b} m_{c}=\frac{3}{2} \sum a^{2}-2 \sum m_{b} m_{c}
$$

hence, by Lemmas 2.1 and 2.6, we have

$$
\begin{aligned}
& \sum\left(r_{b}-r_{c}\right)^{2}-k \cdot \sum\left(m_{b}-m_{c}\right)^{2}-2\left(r_{1}-m_{1}\right)^{2}+2 k\left(m_{1}-m_{2}\right)^{2} \\
& \quad=2\left[\sum r_{a}^{2}-r_{1}^{2}-2 m_{1}^{2}\right]+2 k\left[\sum m_{b} m_{c}-2 m_{1} m_{2}-m_{2}^{2}-\frac{3}{8}(b-c)^{2}\right]
\end{aligned}
$$




$$
\begin{aligned}
& \geq \frac{3 s(s-a)(b-c)^{2}}{2(s-b)(s-c)}-\frac{3 k s(s-a)(b-c)^{2}}{8(s-b)(s-c)} \\
& =\frac{3(4-k) s(s-a)(b-c)^{2}}{8(s-b)(s-c)} \geq 0
\end{aligned}
$$

The proof of Lemma 2.8 is complete.

Lemma 2.9 (see $[4,6,7])$ Define

$$
F(x)=a_{0} x^{n}+a_{1} x^{n-1}+\cdots+a_{n}
$$

and

$$
G(x)=b_{0} x^{m}+b_{1} x^{m-1}+\cdots+b_{m}
$$

If $a_{0} \neq 0$ or $b_{0} \neq 0$, then the polynomials $F(x)$ and $G(x)$ have a common root if and only if

$$
\left.R(F, G):=\left|\begin{array}{ccccccc}
a_{0} & a_{1} & \cdots & a_{n} & & & \\
& a_{0} & a_{1} & \cdots & a_{n} & & \\
& & \ddots & \ddots & & \ddots & \\
& & & a_{0} & a_{1} & \cdots & a_{n} \\
b_{0} & b_{1} & \cdots & b_{m} & & & \\
& b_{0} & b_{1} & \cdots & b_{m} & & \\
& & \ddots & \ddots & & \ddots & \\
& & & b_{0} & b_{1} & \cdots & b_{m}
\end{array}\right|\right\}=0
$$

where $R(F, G)((m+n) \times(m+n)$ determinant $)$ is Sylvester's resultant of $F(x)$ and $G(x)$.

Lemma 2.10 (see $[7,8]$ ) Given a polynomial $f(x)$ with real coefficients

$$
f(x)=a_{0} x^{n}+a_{1} x^{n-1}+\cdots+a_{n}
$$

if the number of the sign changes in the revised sign list of its discriminant sequence

$$
\left\{D_{1}(f), D_{2}(f), \ldots, D_{n}(f)\right\}
$$

is $v$, then the number of the pairs of distinct conjugate imaginary roots of $f(x)$ equals $v$. Furthermore, if the number of non-vanishing members in the revised sign list is $l$, then the number of the distinct real roots of $f(x)$ equals $l-2 v$.

\section{The proof of Theorem 1.1}

Proof If $k \leq 0$,we can easily find that inequality (1.1) holds. Hence, we only need to consider the case $k>0$, and by Lemma 2.7, we only need to consider the case $0<k \leq 4$.

Now we determine the best constant $k$ such that inequality (1.1) holds. Since inequality (1.1) is symmetrical with respect to the side-lengths $a, b$ and $c$, there is no harm in supposing $a \leq b \leq c$. Thus, by Lemma 2.8 , we only need to determine the best constant $k$ such 
that

$$
2\left(r_{1}-m_{1}\right)^{2}-2 k\left(m_{1}-m_{2}\right)^{2} \geq 0
$$

or, equivalently, that

$$
\begin{aligned}
& \left(\frac{a \sqrt{(b+c)^{2}-a^{2}}}{2(b+c-a)}-\frac{\sqrt{(b+c)^{2}-a^{2}}}{2}\right)^{2}-k\left(\frac{\sqrt{(b+c)^{2}-a^{2}}}{2}-\frac{1}{2} \sqrt{2 a^{2}+\frac{1}{4}(b+c)^{2}}\right)^{2} \\
& \geq 0
\end{aligned}
$$

Without loss of generality, we can assume that

$$
a=t \quad \text { and } \quad \frac{b+c}{2}=1 \quad(0<t \leq 1)
$$

because inequality (3.1) is homogeneous with respect to $a$ and $\frac{b+c}{2}$. Thus, clearly, inequality (3.1) is equivalent to the following inequality:

$$
\left(\frac{t \sqrt{4-t^{2}}}{2(2-t)}-\frac{\sqrt{4-t^{2}}}{2}\right)^{2}-k\left(\frac{\sqrt{4-t^{2}}}{2}-\frac{\sqrt{2 t^{2}+1}}{2}\right)^{2} \geq 0 .
$$

We consider the following two cases separately.

Case 1. When $t=1$, inequality (3.2) holds true for any $k \in R:=(-\infty,+\infty)$.

Case 2. When $0<t<1$, inequality (3.2) is equivalent to the following inequality:

$$
k \leq \frac{4(2+t)\left(\sqrt{4-t^{2}}+\sqrt{2 t^{2}+1}\right)^{2}}{9(2-t)(1+t)^{2}} .
$$

Define the function

$$
g(t):=\frac{4(2+t)\left(\sqrt{4-t^{2}}+\sqrt{2 t^{2}+1}\right)^{2}}{9(2-t)(1+t)^{2}}, \quad x \in(0,1)
$$

Calculating the derivative for $g(t)$, we get

$$
g^{\prime}(t)=\frac{8\left(\sqrt{4-t^{2}}+\sqrt{2 t^{2}+1}\right) \cdot \sqrt{4-t^{2}} \cdot\left[\left(2 t^{3}+5 t^{2}+10 t-2\right)-(2-t) \sqrt{4-t^{2}} \cdot \sqrt{2 t^{2}+1}\right]}{9(2-t)^{2}(1+t)^{3} \sqrt{2 t^{2}+1} \cdot \sqrt{4-t^{2}}} .
$$

By setting $g^{\prime}(t)=0$, we obtain

$$
\sqrt{4-t^{2}} \cdot\left[\left(2 t^{3}+5 t^{2}+10 t-2\right)-(2-t) \sqrt{4-t^{2}} \cdot \sqrt{2 t^{2}+1}\right]=0 .
$$

It is easily observed that the equation $\sqrt{4-t^{2}}=0$ has no real root on the interval $(0,1)$. Hence, the roots of equation (3.4) are also solutions of the following equation:

$$
\left(2 t^{3}+5 t^{2}+10 t-2\right)-(2-t) \sqrt{4-t^{2}} \cdot \sqrt{2 t^{2}+1}=0
$$

that is,

$$
(1+t)^{2} \varphi(t)=0,
$$


where

$$
\varphi(t)=t^{4}+10 t^{2}-2
$$

It is obvious that the equation

$$
(1+t)^{2}=0
$$

has no real root on the interval $(0,1)$.

It is easy to find that the equation

$$
\varphi(t)=0
$$

has one positive real root. Moreover, it is not difficult to observe that $\varphi(0)=-2<0$ and $\varphi(1)=9>0$. We can thus find that equation (3.7) has one distinct real root on the interval $(0,1)$. So that equation (3.4) has only one real root $t_{0}$ given by $t_{0}=0.442890982868958 \ldots$ on the interval $(0,1)$, and

$$
g(t)_{\max }=g\left(t_{0}\right) \approx 3.2817755127 \in(3,4) .
$$

Now we prove $g\left(t_{0}\right)$ is the root of equation (1.2). For this purpose, we consider the following nonlinear algebraic equation system:

$$
\left\{\begin{array}{l}
\varphi\left(t_{0}\right)=0, \\
2 t_{0}^{2}+1-u_{0}^{2}=0, \\
4-t_{0}^{2}-v_{0}^{2}=0, \\
4(2+t)\left(u_{0}+v_{0}\right)^{2}-9(2-t)(1+t)^{2} k=0 .
\end{array}\right.
$$

It is easy to see that $g\left(t_{0}\right)$ is also the solution of nonlinear algebraic equation system (3.9). If we eliminate the $v_{0}, u_{0}$ and $t_{0}$ ordinal by the resultant (by using Lemma 2.9), then we get

$$
29,648,323,021,629,456 \cdot \phi_{1}^{2}(k) \cdot \phi_{2}^{2}(k)=0,
$$

where

$$
\phi_{1}(k)=6,561 k^{4}-14,256 k^{3}-18,080 k^{2}-25,344 k+20,736
$$

and

$$
\phi_{2}(k)=729 k^{4}-7,344 k^{3}+8,800 k^{2}-13,056 k+2,304 .
$$

The revised sign list of the discriminant sequence of $\phi_{1}(k)$ is given by

$$
[1,1,-1,-1]
$$


The revised sign list of the discriminant sequence of $\phi_{2}(k)$ is given by

$$
[1,1,-1,-1]
$$

So the number of sign changes in the revised sign list of (3.11) and (3.12) are both 2. Thus, by applying Lemma 2.10 , we find that the equations

$$
\phi_{1}(k)=0
$$

and

$$
\phi_{2}(k)=0
$$

both have two distinct real roots. In addition, it is easy to find that

$$
\begin{array}{ll}
\phi_{1}(0)=20,736>0 ; & \phi_{2}(0)=2,304>0, \\
\phi_{1}(1)=-30,383<0 ; & \phi_{2}(1)=-8,567<0, \\
\phi_{1}(3)=-71,487<0 ; & \phi_{2}(8)=-313,088<0
\end{array}
$$

and

$$
\phi_{1}(4)=397,312>0 ; \quad \phi_{2}(9)=26,793>0 .
$$

We can thus find that equation (3.13) has two distinct real roots on the intervals

$$
(0,1) \text { and }(3,4) \text {. }
$$

And equation (3.14) has two distinct real roots on the intervals

$$
(0,1) \text { and }(8,9) \text {. }
$$

Hence, by (3.8), we can conclude that $g\left(t_{0}\right)$ is the root of equation (1.2). The proof of Theorem 1.1 is thus completed.

Competing interests

The authors declare that they have no competing interests.

Authors' contributions

Both authors contributed equally and read and approved the final manuscript.

\section{Author details}

'Department of Education, Zhejiang Teaching and Research Institute, Hangzhou, Zhejiang 310012, People's Republic of China. ${ }^{2}$ Department of Mathematics, Zhejiang Xinchang High School, Shaoxing, Zhejiang 312500, People's Republic of China.

\section{Acknowledgements}

The authors would like to thank the anonymous referees for their very careful reading and making some valuable comments which have essentially improved the presentation of this paper. 


\section{References}

1. Liu, B-Q: BOTTEMA, What We Have Seen - the New Theory, New Method and New Result of the Research on Triangle. Tibet People Press, Lhasa (2003) (in Chinese)

2. Yang, L: A dimension-decreasing algorithm with generic program for automated inequality proving. High Technol. Lett. 25(7), 20-25 (1998) (in Chinese)

3. Yang, L: Recent advances in automated theorem proving on inequalities. J. Comput. Sci. Technol. 14(5), $434-446$ (1999)

4. Yang, L, Xia, B-C: Automated Proving and Discovering on Inequalities. Science Press, Beijing (2008) (in Chinese)

5. Yang, L, Xia, S-H: Automated proving for a class of constructive geometric inequalities. Chinese J. Comput. 26(7), 769-778 (2003) (in Chinese)

6. Sylvester, JJ: A method of determining by mere inspection the derivatives from two equations of any degree. Philos. Mag. 16, 132-135 (1840)

7. Yang, L, Zhang, J-Z, Hou, X-R: Nonlinear Algebraic Equation System and Automated Theorem Proving, pp. 23-25. Shanghai Scientific and Technological Education Press, Shanghai (1996) (in Chinese)

8. Yang, L, Hou, X-R, Zeng, Z-B: A complete discrimination system for polynomials. Sci. China Ser. E 39(7), 628-646 (1996)

doi:10.1186/1029-242X-2013-329

Cite this article as: Shi and Wu: An artificial proof of a geometric inequality in a triangle. Journal of Inequalities and Applications 2013 2013:329.

\section{Submit your manuscript to a SpringerOpen ${ }^{\circ}$ journal and benefit from:}

- Convenient online submission

- Rigorous peer review

Immediate publication on acceptance

- Open access: articles freely available online

- High visibility within the field

- Retaining the copyright to your article 